\title{
Diagnostic Assessment of Movement Disorders and Psychomotor Deficiency in Residual Schizophrenia
}

\author{
Vera Ilanković ${ }^{1}$, Andrej N. Ilanković ${ }^{2}$, Nikola N. Ilanković ${ }^{3^{*}}$ \\ ${ }^{1}$ Faculty of Special Education and Rehabilitation, University of Belgrade, Belgrade, Serbia; ${ }^{2}$ Department of Organic Mental \\ Disorders, Center of Clinical Neurophysiology \& Sleep, Research, Psychiatric Clinic, Clinical Center of Serbia, Belgrade, \\ Serbia; ${ }^{3}$ University of Belgrade, Medical Faculty, Belgrade, Serbia
}

\begin{abstract}
Citation: llanković V, llanković AN, llanković NN. Diagnostic Assessment of Movement Disorders and Psychomotor Deficiency in Residual Schizophrenia. OA Maced J Med Sci 2014 Jun 15; 2(2):239-243. http://dx.doi.org/ 2014.038

Key words: schizophrenia residual type movement disorders; psychomotor deficiency; clinical assessment.

"Correspondence: Nikola N. Ilanković Psychiatric Clinic, Clinical Center of Serbia, Belgrade, Pasterova 2. E-mail: nikolanilankovic@gmail.com

Received: 13-Jan-2014; Revised: 26-Feb2014; Accepted: 05-Mar-2014; Online first: 02-Apr-2014

Copyright: () 2014 llanković et al. This is an open-access article distributed under the terms of the Creative Commons Attribution License, which permits unrestricted use, distribution, and reproduction in any medium, provided the original author and source are credited.

Competing Interests: The authors have declared that no competing interests exist.
\end{abstract}

\begin{abstract}
Movement disorders and motor deficiency is highly represented among patients in Schizophrenia residual type (DSM IV): $37 \%$ of patients examined in our research had psycho-motor disturbances. Dominant disturbances are the following: disturbed tonus among $65 \%$ of patients, abnormal postural reactions among $90 \%$, abnormal voluntary movements among $75 \%$, disturbed speech among $85 \%$, disturbed static and dynamic postures among $90 \%$. Involuntary movements are present among $20 \%$ of patients.

Classic neurological diagnostics offers general assessment of the motor status. Standardized clinical motor scales only register the presence and intensity of the motor disturbance. Rehabilitation treatment requires individual and up to date functional diagnostics of the motor deficiency.

We would like to stress that psychiatric patients in psychotic, (prepsychotic) and postpsychotic states, besides being mentally ill, also suffer from serious movement disorders (psychomotor disturbances and deficits) which put them into the category of disabled persons, who besides requiring psychiatric (neuropsychiatric) treatment, also require systematic psychomotor and speech rehabilitation.
\end{abstract}

\section{Introduction}

Following-up the historical development of clinical psychiatry in reference with descriptions of clinical symptomatology/phenomenology of mental illnesses (psychoses), one notes that authors of the first period attach prime importance to psycho-pathological phenomena, to general descriptions of behavioral disturbances and the psycho-motor field, and, thus, to social consequences of the altered behavior of patients.

It is only considerably later that neurological and cognitive disturbances were being noted in acute and especially chronic (residual, "negative") forms of so-called "functional" (endogenous) psychotic disturbances from the field of schizophrenia, but also in recidive and chronic forms of affective and delusional psychoses.

It seems that in spite of the fact that cognitive
disturbances which are now thanks to
neuro-psychological, neuro-physiological and

"neuro-image" techniques (visualization techniques) currently undergoing a research trend, the field of research of motor (psycho-motor) disturbances and deficiensis is still being neglected and brought down to general descriptions of "bizarre" body posture, movement and behavior, without more precise diagnostics and appropriate treatment being applied.

\section{Motor Disturbances in Schizophrenia}

Summing up classic studies of motor disturbances in schizophrenia one can note that the clinical approach oscillates in phases between "cerebral" (neurological) and "non-cerebral" (psychological) means of explaining these phenomena. It is impressive how precisely such prominent names of clinical psychiatry (Kraepelin, Kahlbaum, Jaspers) describe motor disturbances, only to much too easily neglect them or rank them as secondary. E. Bleuler who said that his patients suffering from schizophrenia were 
"recognized by the way they walked and their posture", on the other hand, mainly reviewed psycho-motor disturbances merely from a psycho-dynamic point of view $[1,4,10]$.

Almost dismissal is, however, most evident in the most advanced classification systems of mental illnesses (DSM-III-R, DSM-IV, ICD-10), which completely obscure a whole spectre of psycho-motor disturbances and speech disturbances in schizophrenia illness! [3, 12].

The beginning of the new so-called psycho-pharmacological era of psychiatry encourages in an unusual way only biochemical research of pathogenesis of endogenous psychosis (influence of the pharmacological industry?), and shows maximum simplification of the clinical approach to ethio-pathogenesis and differential diagnosis of mental disturbances based on the principle "if there is a cure, the cause is not important...". On the other hand, complications which appear in the application of neuroleptic medication (motor disturbances of the following type: acute dyskinesia, medicamentous Parkinsonism and tardive dyskinesia), again focuses the attention of people working in clinics on motor disturbances, although not for reasons of reviewing the basic nature of the illness, but due to fear of side effects (and high insurance claims, for instance in the USA) $[2,11,12]$. At the same time, thanks to "chemical humanization" of the environment in mental hospitals, there is a tendency of maximum development of the psycho-social approach in the treatment and rehabilitation of patients suffering from schizophrenia and other mental illnesses.

It was only in the eighties that Theo Manschreck and his colleagues in Boston started focusing attention again to motor disturbances in schizophrenia producing a series of clinical studies noting down the following data: generalized lack of coordination and clumsiness, movement and posture stereotypes (as rubbing, nodding, facial gestures), mannerism, motor actions blocks, disturbances of voluntary activities [6, 7]. Motor abnormalities were associated with: association disturbances, supine affects and other so-called "soft neurological signs".

Capital contribution to this field of research was given by the British neurologist and psychiatrist Daniel Rogers who in 1978 furthered the pioneer work of Richard Hunter. He produced a brilliant retrospective study covering the period of time from 1911 (when E. Bleuler introduced the notion "Schizophrenia") to 1955, i.e. up to the "neuroleptic era", and after 1955 (1955-1978) i.e., the era of massive application of neuroleptics $[8,9]$.

\section{Rogers' work showed [8]:}

- That $88 \%$ of persons suffering from schizophrenia had at least one motor (neurological) disturbance even prior to their first admission to a psychiatric hospital!
- That $92 \%$ of the patients had motor disturbances during the first 5 years of hospitalization even without undergoing any biological treatment,

- That $98 \%$ of persons suffering from schizophrenia had at least one type of motor deficiency before 1955, i.e. before the introduction of neuroleptic therapy!

- That all $100 \%$ of persons suffering from schizophrenia examined in 1992 had at least one type of motor deficiency, and $91 \%$ had more categories of motor disturbances!

Today in clinical psychiatry develop the new tendency to assess the neuropsychological (cognitive) impairment and the so called soft neurological signs. [13]

\section{Assessment of Motor Deficiency in Schizophrenia}

We based our review of motor deficiency in chronic psychotic condition (Schizophrenia - residual type) on one theoretical framework and three empirical argumentations.

The theoretical framework of our approach to the assessment of motor (psycho-motor) disturbances of schizophrenic patients based on the theory of the dissolution and resolution theory of nervous system and evolutionary hierarchy system of function in the nervous system (Jackson H, 1834, 1911) [1, 5].

The empirical, practical argumentation of our approach to the assessment and treatment of motor (psycho-motor) deficiency in residual schizophrenia is based on personal experience in diagnostics and therapeutic work with four population groups of patients:

1. working with children suffering from cerebral palsy, i.e. from different forms and degrees of psycho-motor retardation;

2. working with injured persons who had suffered difficult cranio-cerebral traumas whose recovery fluctuated over different levels of consciousness disturbances and psycho-motor regression;

3. working with persons suffering from acute psychosis manifesting most severe forms of functions dissolution i.e., psychic and motor regression; and finally;

4. working with persons suffering from chronic residual psychosis of the schizophrenia type who, unfortunately, besides partially correctable psycho-motor disturbances, also suffer from permanent motor/psycho-motor deficiencies (which along with mental disturbances significantly increase their degree of disability - handicap!)

The common denominator of the majority of these ill conditions in the clinical and pathophysiological 
sense of the word is deep psycho-motor regression which manifests itself through: pathological reflex activity; pathological tonus; lack of adequate postural reflex activity; pathological body posture; pathological movement; and impoverished movement.

\section{General Diagnostic Methods}

General assessment of motor (psycho-motor) deficiency includes: 1) detailed anamnesis; 2) clinical neurological examination; 3) consultative and complementary examinations; and 4) functional status.

Classic neurological examination offers minimal data on motor functioning of the patient. That fact imposes the necessity for detailed functional assessment of the condition of the locomotor system by means of physio-therapeutic diagnostic methods pertaining to: - assessment of the level of motor regression by the "Reflex Activity Test" (llankovic $\mathrm{V}$ et llankovic N, 1995[12]); - assessment of the existence of normal postural reactions (reactions of straightening up, tilting and balance); - assessment of the existence of pathological postural reactions (reactions of straightening up, tilting and balance); - registering the existence of muscular elasticity disturbances (contractures, hypermobility); - registering muscular power disturbances (general); - registering congenital deformations of the bone joint system; - registering of acquired deformations of the bone joints system; assesment of communication capacity of patient.

\section{Specific Measuring Scales - Rating Scales for Motor Disturbances}

For detailed assessment of motor disturbances quantified assessment scales (rating scales) are used specified for certain qualities, types and degrees of manifestation of particular motor dysfunction or/and deficiencies [10]. We choose as the most appropriate the following rating scales for motor disturbances in psychiatry (we applied them for diagnostics and evaluation of the results of the applied treatment as well):

1. Abnormal Involuntary Movement Scale AIMS,

2. Depressive Retardation Rating Scale - DRS,

3. Praxia Examination Protocol,

4. Left Right Orientation Test,

5. Simultaneous Movement Test TSP [10, 14 15].

Our research subject was psychomotor deficiency and movement disorders of chronic mentally ill patients suffering from schizophrenia - residual type (according DSM IV).

The basic idea of our research was to point out the possibility of diagnostic pertaining to these very disabling disturbances as well as to offer an early rehabilitation program which will alleviate the degree of disability of these very young patients $[15,16]$.

The aim of the study was objective assessment of the functional motor status of patients suffering from chronic psychotic disturbances: Schizophrenia (residual type).

\section{Material and Methods}

\section{Specimen Groups}

Group "1" Schizophrenia residual type: 60 persons examined, $M$ and $F$ sex, between 30 and 45 years of age, clinically diagnosed as suffering from schizophrenia - residual type (according to DSM-IV), with no data on: infections, drug abuse and craniocerebral injuries.

The structure of the group was: according to sex - Men 30 or $50 \%$, Women 30 or $50 \%$; according to diagnosis: 100\% dg: Schizophrenia - residual type (DSM-IV); and according to age: minimum 30 years of age, maximum 45 years of age.

\section{Time and place of research}

The complete research was implemented at the Psychiatric University Clinic in Belgrade Belgrade/Serbia in the period of time between 2010 and 2012.

\section{Instruments and Methods}

General questionnaire for patients or "Questioner for assessment of motor deficiency and treatment evaluation" Contains basic personal and clinical data about the patients examined and a joint breakdown of individual items of all the particular questioners/measuring scales for the assessment of motor deficiency.

Special questionnaires/clinical measuring scales were also used (see Specific Measuring Scales Rating Scales for Motor Disturbances)

NOTE: The evaluation was always done by two independent examiners - qualified special educator and physician specialist - neuropsychiatrist.

\section{Results and Discussion}

Results on the Involuntary Movement Scale (AIMS)

The Involuntary Movement Scale (AIMS) which assisted us in registering involuntary movements of various location (face and mouth, extremities, torso), regardless of their origin (ethiopathogenesis), indicated relatively high presence of these pathological motor phenomena in the examined population.

Patients have been registered to have an 
average pathological score of 9.51 .

\section{Results on the Depressive Retardation Scale}

Depressiveness among the majority of psychiatric patients, as well as in residual psychotic conditions represents a highly significant factor and generator of psychopathology, pathoplastic and one of dominant causes of psychological and psychomotor regression which can considerably deteriorate the clinical picture of the illness, as well as the cognitive and motor performance of ill persons! The reduction of depressiveness (including schizophrenic patients) significantly contributes to the improvement of the subjective and objective condition of the patient, but also paves the way for optimal cooperation and good results in the therapeutic and rehabilitation process.

The Depressive Retardation Scale which we applied in our research indicated that a I I patients examined showed an exceptionally high degree of depressive retardation - the average score was as high as 54.23! (The maximum score is 60 , and the score of $>20$ is already marked as depressive retardation!)

\section{Results on the Left-Right Orientation Test}

During our nearly three-year long work with residual psychosis, but also with acute psychotic decompensation, we noted serious disturbances - in body schemes, space orientation of parts of the body, recognition of parts of body, left-right orientation. We expected prominent $L-R$ disorientation in residual conditions as well, but our research produced quite opposite results. The results indicate that the average value achieved at the test was 6.73 of maximum 8 .

\section{Results on Praxia Protocol}

Patients suffering from acute schizophrenia showed in our research a noticeable high level of deterioration of the most complex psycho-motor functions demanded in praxia tests! Patients have especially large problems when trying to perform complex movements and highly coordinated actions, as well as in ideation and initiation of the same.

The important problem is missed time anticipated for timely, early rehabilitation, so that these patients, besides their basic psycho-motor deficiency, have already formed pathological postural patterns, fixed pathological body postures, often acquired deformations of the bone-joint structure system and bad habits in dynamic posture and gestures (prepsychotic origin?, early neurodevelopmental damages?).

The overall achievement on the "Praxia Protocol" among the patients we researched was within the middle value of 30.90 of the maximum possible 75 , i.e., only $41 \%$.

Results on the Simultaneous Movement Test (TSM)
The Simultaneous Movement Test (llankovic V [14]) showed good sensibility towards vitally significant psycho-motor activities of patients suffering from acute psychosis. A man has a pair of upper and lower extremities which thanks to complex neural mechanisms of regulation and integration can be used unilaterally synchronous, unilaterally asynchronous, bilaterally synchronous, bilaterally asynchronous, and reciprocally. Within our specimen group we noticed that the patients examined had the most difficulties performing complex movements. This was particularly manifest in cases where patients were supposed to perform simultaneously two different complex movements simultaneously with both hands - this our patients found practically impossible to achieve!

The overall achievements on the Test were for this reason very poor - the average achievement before treatment was 1.97 (of max. possible 9) i.e. 21\%.

In conclusion: 1) psychomotor deficiency is highly represented among patients suffering from chronic psychotic disorder (Schizophrenia-residual type) $-100 \%$ of the patients examined in our research had serious psycho-motor disturbances. $37 \%$ of patients examined in our research had psycho-motor disturbances. Dominant disturbances are the following: disturbed tonus among $65 \%$ of patients, abnormal postural reactions among $90 \%$, abnormal voluntary movements among $75 \%$, disturbed speech among $85 \%$, disturbed static and dynamic postures among $90 \%$. Involuntary movements are present among $20 \%$ of examined patients; 2) classic neurological diagnostics enables merely a general assessment of the motor status. Standardized clinical motor scales merely register the presence and intensity of the motor disturbance. Adequate pharmacotherapy and early rehabilitation treatment requires individual and updated functional diagnostics of the psychomotor and speech deficiency, too. Based on the theoretical postulates of Jacksonism, we modified and complemented the existing diagnostics battery with the Reflex activity test aimed at assessing the level of motor regression (dissolution of functions) and with the Simultaneous movements test (TSP); and 3) the general conclusion is that the so called psychiatric patients besides being mentally ill, also suffer from serious psychomotor and speech disturbances, which also classify them into the category of physically ill and handicapped persons. It is evident that these patients besides neuropsychiatric treatment require a systemic and early rehabilitation treatment as well.

\section{References}

1. Kaplan HI, Sadock BJ, Grebb JA. Synopsis of Psychiatry, 7th Edition. Williams \& Wilkins: Baltimore, 1994.

2. Ilankovic N, llankovic V. The Restorative Psychiatry, VIII World Congress of Psychiatry, Athens, 1989.

3. American Psychiatric Association. Diagnostic and Statistic Manual of Mental Disorders (DSM-III-R), 3rd ed. Revised APA, 
1987.

4. Crow TJ. The Two-syndrome Concept: Origins and Current Status, Schizophr. Bull. 1985;11:471.

5. Ilankovic N, Jasovic-Gasic M, Jasovic A. [Cerebral Pathology and Psychosis], Engrami. 1988; 1-2:19-25.

6. Manschreck $T$ et al. Disturbed Voluntary Motor Activity in Schizophrenic Disorder. Psychological Medicine. 1982;12:73-84.

7. Manschreck T et al. Psychopathology of Motor Behaviour in Schizophrenia. Progr In Exp Personality Research. 1983; 12:53-99.

8. Rogers $D$ et al. The Motor Disorders of Severe Psychiatric Illnesses: A Conflict of Paradigms. British Journal of Psychiatry. 1985;142:1471-4.

9. Rogers $\mathrm{D}$ et al. The Motor Disorder of Mental Handicap: An Overlap With Motor Disorders of Severe Psychiatric IIInesses. British Journal of Psychiatry. 1991;158:97-102.

10. Timotijevic I, Paunovic VR. [Instruments of Clinical Assessment in Psychiatry]. Naucna Knjiga: Beograd, 1992.

11. Ilankovic $\mathrm{N}$ et al. [Tardive Dyskinesia (TD) - Prevention, Diagnostics, Treatment]. Engrami. 1990; 1-2.

12. Ilankovic V, Ilankovic N. [Schizophrenia - An Illness of the Soul and/or Body - Motor Deficiency in Schizophrenia, Assessment and Treatment]. CIBIF - Medicinski fakultet: Beograd, 1995.

13. Boks MP, Liddle PF, Burgerhof JG, Knegtering R, Bosch RJ. Neurological soft signs discriminating mood disorders from first episode schizophrenia. Acta Psychiatr Scand. 2004; 110(1):2935.

14. Ilankovic V, llankovic N. Restorativna kineziterapija (4. izdanje). Medicinski fakultet - Fasper, Univerzitet u Beogradu: Beograd, 2013.

15. Ilankovic N, llankovic V. Restaurativna psihijatrija. Medicinski fakultet: Beograd, 2001.

16. Ilankovic V, llankovic N. Psihomotorni razvoj deteta (4. izdanje). Medicinski fakultet - Fasper, Univerzitet u Beogradu: Beograd, 2013. 